\title{
Investigação anatomopatológica do sistema respiratório inferior após inoculação fúngica em roedores imunossuprimidos
}

\author{
Priscila Damasceno Abadio ${ }^{1}$ \\ Marcia Silva de Oliveira ${ }^{2}$
}

\begin{abstract}
RESUMO - O progresso médico-científico tornou rotineiro o número de procedimentos invasivos, juntamente com o uso de antibióticos e terapêuticas imunossupressoras. Isso acarretou uma série de novas patologias e obstáculos a serem pesquisados. $\mathrm{O}$ Aspergillus é um fungo oportunista que se destaca entre os demais agentes patológicos apresentando grande incidência de casos relatados na literatura médica, agindo principalmente em pacientes imunocomprometidos e afetando grande parte do sistema respiratório inferior (pulmões). A ausência de resultados histológicos especulada por Latgé deve-se à dificuldade de um modelo experimental ideal, tornando, além dos objetivos reais dessa pesquisa, o estudo como crítica ao modelo já criado.
\end{abstract}

Palavras-chave: Aspergillus fumigatus, pulmão, dexametasona, munossupressão.

\section{Anatomopatologic investigation of the inferior respiratory system after fungic inoculation in imunossuppressed rodents}

\begin{abstract}
The scientific medical progress became common the number of invasive procedures with the use of antibiotics and therapeutical imunossuppression. This revolution caused a series of new patology and obstacles to be searched. The Aspergillus is an opportunist fungi that it has detached amongst oders pathological agents, acting mainly in imunocompromised affecting and patients, over all, great part of the low respiratory system: the lungs. The absence of histologic results is speculated by Latgé, due to difficulty of finding an ideal experimental model, becoming beyond the real objectives of this research, the study, as a critic to the model already created.
\end{abstract}

Key words: Aspergillus fumigatus, lung, dexamethasone, imunossupression.

\footnotetext{
${ }^{1}$ Aluna do curso de graduação em Ciências Biológicas do Centro Universitário de Brasília-UniCEUB. E-mail: priscilaabadio@yahoo.com.br

${ }^{2}$ Professora dos cursos de graduação em Biomedicina e Biologia do Centro Universitário de Brasília UniCEUB.E-mail:professora_df@brturbo.com.br
}

Univ. Ci. Saúde, Brasília, v. 3, n. 2, p. 313-327, jul./dez. 2005 
Os pulmões são órgãos leves e esponjosos localizados na cavidade torácica, responsáveis pela respiração e oxigenação sangüínea. Possuem uma área de superfície de troca de, aproximadament,e $100 \mathrm{~m}^{2}$, apresentando extraordinária capacidade de interação entre os meios interno e externo ${ }^{1}$.

Existem, nos pulmões, de acordo com o relatado por Filho (2000), células especializadas em auxiliar a defesa contra agentes patogênicos presentes nos órgãos (células ciliadas, mucossecretoras, células de clara, células do sistema imunitário), que agem retirando o agente agressor retido. Mesmo assim, é possível detectar microorganismos em diferentes níveis da árvore brônquica ao formar colônias que, nem sempre, produzem reação patogênica ao indivíduo, criando grande dilema em relação à conduta da infecção hospitalar².

Nas últimas décadas, as infecções fúngicas têm promovido estudos e discussões em razão do aumento de quadros infecciosos hospitalares. Foi possível constatar que isso ocorre pelo número de procedimentos invasivos realizados nos pacientes, tratamentos quimioterápicos, terapêutica imunossupressora, patologias imunossupressoras e aumento do uso de antibióticos ${ }^{3}$. De acordo com Marchiori (2002; 2003), as alterações patológicas pulmonares provocadas pelo fungo Aspergillus apresentam-se variadas e dependentes do estado imunológico e das condições pulmonares do paciente, provocando uma gama de patologias (além de pneumonia), sendo possível observar, em cortes histológicos do pulmão, comprometimentos que abrangem os brônquios, bronquíolos e alvéolos pulmonares ${ }^{4}$.

O Aspergillus fumigatus é um fungo saprofítico, oportunista que se tem destacado pela sua incidência e seu nível de mortalidade em pacientes imunodeprimidos ${ }^{5}$. Possui formato filamentoso ${ }^{6}$, micélios vegetativos com hifas hialinas septadas que variam de 3 ìm a 12ìm de acordo com Lopes (2004). São fungos bastante difundidos no meio ambiente, apresentando-se no solo, na água, em matérias orgânicas e em decomposição. A facilidade de infectar-se vem da simplicidade de encontrá-lo e, principalmente, por propagar-se pelas correntes de ar, podendo alojar-se e multiplicar-se em outras áreas .

Os esporos do Aspergillus geram infecção dependendo das condições imunológicas do indivíduo. De acordo com esse aspecto, o fungo pode-se comportar como sensibilizante, colonizador ou invasor, gerando diferentes quadros clínicos

\footnotetext{
${ }^{1}$ MOORE et al, 1998; DI DIO et al, 2002.

${ }^{2}$ FILHO, 2000.

${ }^{3}$ NEVES et al, 2004.

${ }^{4}$ JHAYYA e cols., 2000.

${ }^{5}$ LATGÉ, 1999; NEVES et al., 2004.

${ }^{6}$ AMORIM e cols., 2004.

${ }^{7}$ LOPES e cols., 2004.
} 
como aspergilose alérgica, colonização intracavitária e aspergilose invasiva ${ }^{8}$. Amorim (2004) relata que os principais fatores responsáveis pela patogenicidade do Aspergillus estão relacionados ao pequeno tamanho de seu conídio facilitando sua dispersão pelo meio, por aspiração e inflamação dos seios paranasais e da arvore brônquica. Também por sua temperatura de crescimento estar na faixa dos $37^{\circ} \mathrm{C}$, apresenta capacidade de aderir-se ao endotélio e ao epitélio, por conseguir invadir os vasos sanguíneos e produzir toxinas, como a elastase (que cliva inibidores de proteases leucocitárias e componentes do complemento), a fumigatoxina entre outras ${ }^{9}$.

A aspergilose é uma doença infecciosa não contagiosa, provocada normalmente pela espécie A. fumigatus. A infecção tem início pela aspiração de conídios presentes no ar. Os casos mais graves de aspergilose relacionados ao pulmão ocorrem pela imunodeficiência de pacientes em razão de terapias imunossupressoras, podendo ser classificados como infecções fúngicas oportunistas $^{10}$.

A espécie Aspergillus fumigatus apresenta três importantes quadros clínicos, especificados por Peixinho (2003), relacionados ao sistema respiratório inferior: a aspergilose broncopulmonar alérgica, a aspergilose pulmonar invasiva e o aspergiloma. O diagnóstico é feito por meio do isolamento do agente nas secreções brônquicas e no tecido pulmonar.

Na aspergilose broncopulmonar alérgica, a colônia de Aspergillus não invade o tecido pulmonar, mas permanece como saprófito na luz das vias aéreas, com isso, os brônquios passam a ser preenchidos por muco contendo elementos fúngicos e eosinófilos, a parede brônquica torna-se espessada pela infiltração celular, e a lesão tecidual determina o surgimento de bronquiectasias ${ }^{11}$. De acordo com Amorim (2004), a aspergilose broncopulmonar alérgica é caracterizada pela reação de hipersensibilidade dos tipos I, III e IV. O processo ocorre quando os esporos inalados chegam aos brônquios de maior calibre, promovendo aumento no número de antígenos, estimulando resposta inflamatória local, e as imunoglobulinas IgE e IgG ativam mastócitos e basófilos, ocorrendo vasodilatação, aumento da permeabilidade vascular e contração da musculatura lisa do brônquio. Isso retém mais esporos do fungo e afluxo de linfócitos. O sistema de complemento também é ativado, aumentando a injúria brônquica.

A Aspergilose pulmonar invasiva é a patologia mais rara e grave, podendo ser localizada na membrana basal das vias aéreas afetando, sobretudo, doentes

${ }^{8}$ LOPES et al., 2004.

${ }^{9}$ NEUFELD, 2004.

${ }^{10}$ AMORIM e cols., 2004.

${ }^{11}$ LATGÉ, 1999; NEUFELD, 2004.

Univ. Ci. Saúde, Brasília, v. 3, n. 2, p. 313-327, jul./dez. 2005 
imunocomprometidos, como, por exemplo, doentes neutropênicos, pacientes infectados pelo HIV e pacientes submetidos à corticoterapia sistêmica. Tarantino (2002) afirma que a aspergilose invasiva se encontra entre as micoses mais comuns adquiridas em hospitais. O fungo cresce no interior dos vasos sanguíneos, gerando hemorragia localizada e áreas de infarto que evoluem para necrose e cavitação; quando crônica, forma colonização intracavitária. Apresenta-se, então, nesse contexto, como um dos problemas mais graves, com alta taxa de mortalidade ${ }^{12}$.

A maioria das infecções causadas por aspergilose encontram-se associadas à terapia imunossupressora e ao período pós-transplante ${ }^{13}$. Para o sucesso do transplante, é necessário que as atividades do sistema imune sejam reduzidas o suficiente para que o órgão não seja rejeitado, mas isso permite a ocorrência de infecções por microorganismos oportunistas ${ }^{14}$.

Os corticosteróides, quando utilizados em altas doses, funcionam como imunossupressores, bloqueando a ação da prostaglandina e reduzindo a atividade de linfócitos e monócitos ${ }^{15}$. A dexametasona é um corticosteróide de longa duração, de uso sistêmico e local, empregado em tratamento sintomático de inflamações crônicas e doenças auto-imunes. Seu principal efeito refere-se à profunda alteração na resposta imune linfocitária, representada pela ação antiinflamatória e imunossupressora, podendo prevenir ou suprimir processos inflamatórios de várias naturezas. Ela age inibindo a enzima fosfolipase A2, responsável pela síntese de prostaglandina, e as funções específicas de linfócitos, entre outras ações.

A presente pesquisa busca simular em roedores (Rattus norvegicus), casos de imunossupressão (pelo uso da droga dexametasona) com conseqüente infecção gerada pela inoculação do fungo Aspergillus fumigatus. Avalia a influência de fatores químicos na manutenção da homeostasia do sistema respiratório e procura determinar que alterações anátomo-fisiológicas, histológicas e patológicas podem advir dessa infecção pela investigação laboratorial do material obtido.

Esse estudo teórico segue uma linha de pesquisa já desenvolvida por alunos de biomedicina do UniCEUB. Tem por finalidade encontrar metodologia eficiente para simular e estudar a aspergilose em laboratório e desenvolver meios de diagnóstico precoce e combate mais eficiente contra essa infecção oportunista.

\footnotetext{
${ }^{12}$ LACAZ et al, 2002; PEIXINHO 2003.

${ }^{13}$ LATGÉ, 1999.

${ }^{14}$ ROITT et al, 2004.

${ }^{15}$ CHAPEL e cols., 2003.
} 


\section{Materiais e Métodos}

Durante o período de tratamento, procedeu-se à inoculação do fungo Aspergillus fumigatus na região peritonial dos roedores, mantendo um grupo de controle. Os roedores foram dissecados de maneira a obterem-se os pulmões para futuras investigações histológicas, sendo feita a observação macroscópica entre os dois grupos e, posteriormente, a preparação técnica do material obtido (histotécnica).

Foram observadas microscopicamente as alterações no grupo imunocomprometido e, posteriormente, correlacionadas ao grupo de controle. Analisaram-se taxas hematológicas e alterações funcionais respiratórias dos pulmões normais com acometimento patológico, discriminando as alterações encontradas decorrentes da patologia inflamatória a que o roedor foi exposto, chegando-se, assim, a uma base de resultados.

De acordo com as observações anatomo-histológicas da patologia foi realizada a revisão bibliográfica, a fim de explicar as conseqüentes alterações decorridas de possível processo inflamatório. A literatura foi analisada e discutida de acordo com as alterações provocadas pela patologia e os resultados encontrados.

A experimentação encontra-se baseada nos princípios éticos adotados pelo Colégio Brasileiro de Experimentação Animal ${ }^{16}$. Os modelos animais foram desenvolvidos a fim de estudar a eficácia de drogas antifúngicas no tratamento da aspergilose ou para avaliar métodos de diagnósticos. Não há nenhum consenso sobre o melhor modelo a usar, ocorrendo a variação entre investigadores não somente com respeito à escolha de um animal (tensão, peso, e sexo) e do regime imunossupressivo (dose, produtos, freqüência das injeções), mas também com respeito ao protocolo do desafio (concentração dos conídios e da rota da injeção). Apesar da heterogeneidade nos modelos animais utilizados, foi possível extrair diversas conclusões ${ }^{17}$. O procedimento descrito foi baseado em Latgé, 1999; Latgé, 2001; Turner et al, 1975 e 1976.

\section{Animais}

Os animais utilizados foram roedores, da linhagem wistar (espécie Rattus norvegicus), todos machos, jovens, no total de 16, devidamente pesados, cedidos pelo biotério do Centro Universitário de Brasília - UniCEUB. Foram divididos

\footnotetext{
${ }^{16}$ COBEA, 1990.

${ }^{17}$ LATGÉ, 1999.
}

Univ. Ci. Saúde, Brasília, v. 3, n. 2, p. 313-327, jul./dez. 2005 
aleatoriamente em quatro grupos distintos contendo quatro roedores em cada um. O grupo I recebeu apenas a solução salina, sendo, portanto o grupo de controle; o grupo II recebeu dexametasona; o grupo III recebeu a solução salina num primeiro momento e, posteriormente, fungo; o grupo IV recebeu dexametasona e fungo.

\section{Exames e análise hematológica}

Foram realizados exames manuais de leucometria e contagem diferencial de leucócitos pela coleta de amostra de sangue com EDTA, retirada da secção da extremidade caudal com bisturi autoclavado e com lâmina descartável. A amostra foi acondicionada em tubos contendo EDTA, e as lâminas preparadas foram coradas com Giemsa para contagem diferencial. Os exames foram realizados em três momentos: no início do experimento (visando avaliar as condições físicas dos animais), após cinco dias de aplicação da dexametasona (observando-se a imunossupressão) e ao final, obtendo-se parâmetros para futura discussão. Após o primeiro exame, foi constatado que ambos os grupos se apresentavam saudáveis, anteriormente ao experimento, tendo permanecido em gaiolas separadas e alimentando-se normalmente com ração e água tratada, diminuindo os riscos de infecção. A contagem total e diferencial de leucócitos faz parte de avaliação de rotina que visa identificar possível interferência, como infecção, neoplasia, alergia ou imunossupressão do paciente. A contagem diferencial mede a percentagem de cada tipo de leucócito presente na amostra ${ }^{18}$.

\section{Isolamento e obtenção do fungo}

Com o auxílio da Micoteca do UniCEUB, foram replicadas colônias de $A$. fumigatus (da coleção numero 004) em meio Agar batata Dextrose (BDA-Merck, produto 1101300500, lote VM150530341), preparado com infusão de 3,9 gramas de BDA para $100 \mathrm{ml}$ de água destilada, tendo sido aquecida e dissolvida em placa aquecedora até obter fervura, distribuídas em respectivos frascos de $10 \mathrm{ml} \mathrm{e}$ autoclavadas. As colônias foram replicadas em seis tubos com crescimento em temperatura ambiente. No período de duas semanas, após o crescimento ideal do fungo, foi possível obter a suspensão de A. fumigatus depois de persistente lavagem com solução fisiológica estéril dos 03 tubos contendo o fungo, sendo que os demais foram descartados em decorrência da contaminação por outro fungo. Os tubos foram lavados, e a solução, transferida para quatro tubos de $40 \mathrm{ml}$ com tam-

\footnotetext{
${ }^{18}$ PAGANA et al, 1998. 
pa, centrifugados por oito minutos a $3.500 \mathrm{rpm}$. O sobrenadante de todos os tubos foi descartado, considerando-se apenas o depósito ao fundo dos tubos, e ressuspendidos com o lavado fúngico. Com o fim da centrifugação do lavado, a cada nova centrifugação, passaram-se dois concentrados para um tubo a fim de chegar-se a único tubo contendo o concentrado. Com o auxílio da câmara de Newbauer, foi possível concluir que havia, aproximadamente, $10^{6}$ conídeos de $A$. fumigatus no concentrado final, tendo sido feito o mesmo cálculo do exame de leucometria. Posteriormente, o concentrado foi reservado na geladeira até o momento da inoculação (dois dias).

\section{Imunossupressão}

A droga utilizada chama-se Decadron® (fosfato sódico de dexametasona), solução injetável $4 \mathrm{mg}$ por $\mathrm{ml}$ (caixa com um frasco-ampola de $2,5 \mathrm{ml}$ ), produzida pela Ache laboratórios farmacêuticos S. A. A dose imunossupressora calculada foi de $0,2 \mathrm{mg} / \mathrm{Kg} / \mathrm{dia}$, tendo sido aplicado $0,1 \mathrm{ml} /$ dia para cada roedor, no período de 28 de março a 06 de abril, ininterruptamente.

\section{Procedimento experimental}

Toda a aplicação de salina (nos grupos I e III) e de dexametasona (nos grupos II e VI) foi subcutânea. Os animais do grupo IV foram inoculados via peritoneal aplicando-se $0,2 \mathrm{mg} / \mathrm{Kg}$ do concentrado fúngico. Toda a técnica teve como fundamento o trabalho Aspergillus fumigatus e Asbpergillosis ${ }^{19}$. No décimo dia, os animais receberam a droga ou a salina e foram sacrificados após 72 horas da inoculação do fungo via peritoneal. O procedimento decorreu, anestesiando-se cada animal com éter, abrindo a cavidade torácica e provocando a hipovolemia ao retirar, em média, de 3 a $5 \mathrm{ml}$ de sangue diretamente do coração; logo após, os animais foram dissecados, extraindo-se o pulmão para futura preparação de lâminas histológicas.

\section{Histotécnica}

Os pulmões foram submetidos a processos de colheita do material, fixação (com formol a 10\%), desidratação e diafanização, para que sua estrutura histológica se mantenha preservada, evitando o processo de degeneração post-mortem. A

\footnotetext{
${ }^{19}$ LATGÉ, 1999.
}

Univ. Ci. Saúde, Brasília, v. 3, n. 2, p. 313-327, jul./dez. 2005 
técnica histológica busca transformar as células e os tecidos em preparações destinadas ao exame microscópio ${ }^{20}$.

\section{Resultados}

Foi possível observar na tabela 1 que os níveis de leucócitos do primeiro exame de leucometria encontram-se normais em relação ao valor de referência, existindo pequena baixa leucocitária nos animais 11 e 16, que, no momento, são desconsideradas em razão do grande nível de estresse. Os valores abaixo do normal são mínimos, e os fatores individuais podem ter contribuído para esse índice. $\mathrm{Na}$ segunda leucometria, observou-se a baixa dos níveis leucocitários dos grupos II e IV. Os resultados do terceiro exame de leucometria foram dispostos no Figura 4 para futuras discussões, demonstrando diferenças estatísticas entre os quatro grupos.

Na contagem leucocitária diferencial, os valores do primeiro exame foram satisfatórios, observando-se que os roedores se encontravam saudáveis ao início dos testes. Os resultados do segundo exame demonstraram linfopenia nos grupos imunossuprimidos com dexametasona. $\mathrm{O}$ terceiro exame apresentou linfopenia mais acentuada, observada no Figura 5. A taxa comparativa entre os monócitos do terceiro resultado também apresentou alterações que se encontram dispostas no Figura 6.

As figuras 1, 2 e 3 são análises de variância de Kruskal-Wallis de sentido único, feitas no programa SigmaPlot 9.0. A análise foi escolhida automaticamente pelo programa, em razão da discrepância entre os valores (alternativa nãoparamétrica). A avaliação macroscópica mostrou-se normal para todos os grupos, com exceção do roedor 07 , que possuía pulmão atrofiado, e do roedor 16, que apresentava pulmão menor que o normal. Na avaliação microscópica observada nas figuras 4, 5 e 6, não foram encontradas alterações relacionadas à patologia discutida, sendo as lâminas confeccionadas e coradas com HE (figura 4 e 5) e Grocott (6). Puderam ser observadas, ao longo do experimento na tabela 1 e na figura 1 , ambos em anexo, a baixa do peso e a dificuldade de locomoção nos roedores do grupo II e IV (imunossuprimidos).

${ }^{20}$ MICHALANY, 2000. 
Investigação anatomopatológica do sistema respiratório

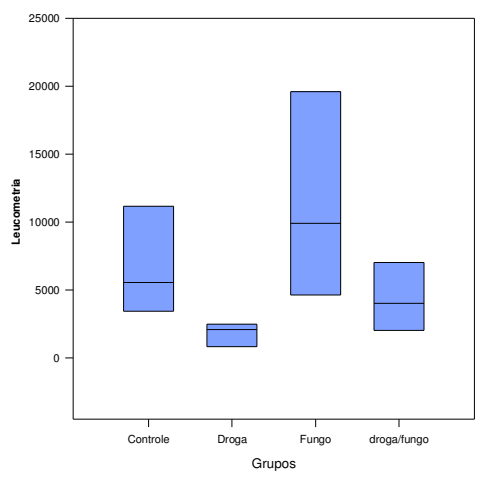

Figura 1 - Comparação da taxa de leucometria total entre os quatro grupos estudados.

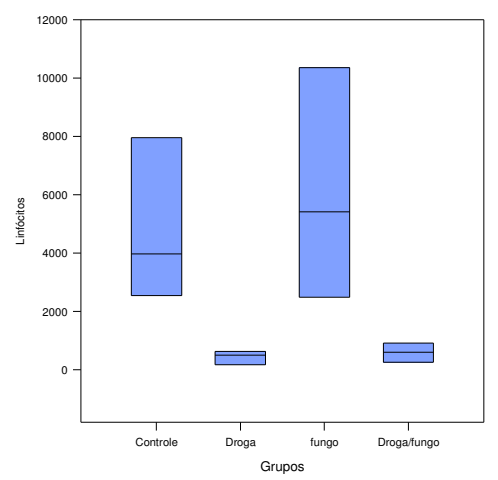

Figura 2 - Mediana de linfócitos: comparação da taxa de linfócitos de hemograma diferencial entre os quatro grupos estudados

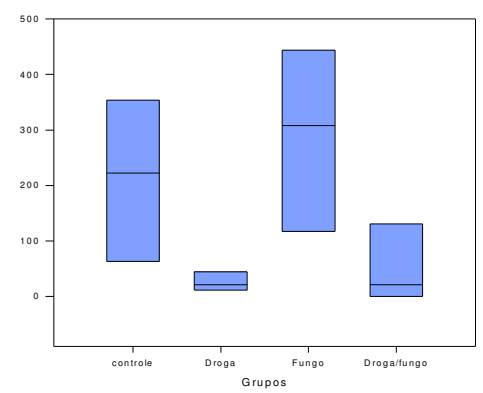

Figura 3 - Mediana de monócitos: comparação da taxa de monócitos de hemograma diferencial entre os quatro grupos estudados.

\footnotetext{
${ }^{21}$ ROINT et al, 2003; 2004.

${ }^{22}$ TARANTINO, 2002.

${ }^{23}$ ROINT et al, 2004.
} 


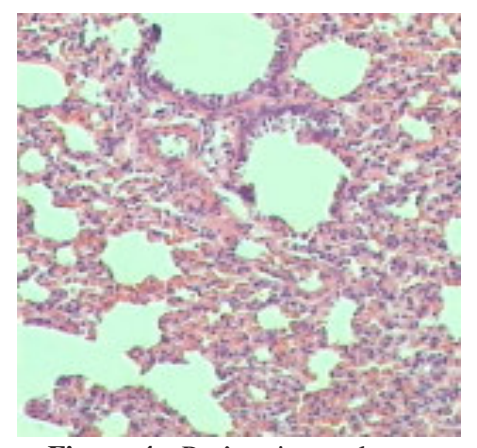

Figura 4 - Parênquima pulmonar - coloração em HE.

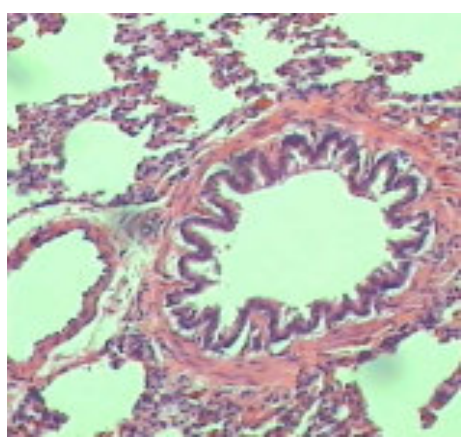

Figura 5 - Bronquíolo - coloração em HE.

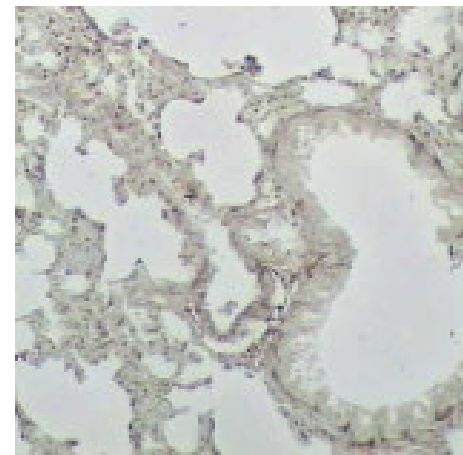

Figura 6 - Parênquima pulmonar - coloração em Grocott

\section{Discussão}

O Aspergillus fumigatus é um patógeno classificado como oportunista estabelecendo-se em indivíduos imunocomprometidos. Nas infecções sistêmicas oportunistas, o mecanismo efetor mais importante é a imunidade mediada por células, sendo a infecção fúngica disseminada, característica de pacientes que apresentam deficiências nas células $\mathrm{T}^{21}$. Um fator relevante é sua termotolerância de $37^{\circ} \mathrm{C}$ a $40^{\circ} \mathrm{C}$, um atributo fundamental para ser considerado patogênico, juntamente com o tamanho de seu conídio que é de fácil dispersão aérea e de tamanho compatível com a deposição alveolar ${ }^{22}$.

\footnotetext{
${ }^{21}$ ROINT et al, 2003; 2004.
}

${ }^{22}$ TARANTINO, 2002. 
O processo inflamatório envolve influxo protetor de leucócitos, sistema de complemento, anticorpos e proteínas plasmáticas no local da infecção. Neutrófilos são ativados ligando-se firmemente à superfície epitelial; macrófagos liberam mediadores, como a citocina, e linfócitos são recrutados para o local de infecção.

A potência antiinflamatória duradoura pode ter gerado aspectos, tais como, a inibição da aderência de neutrófilos ao endotélio vascular em uma áre a inibição da aderência de neutrófilos ao endotélio vascular em uma área inflamatória e a supressão de monócito/macrófago, como a atividade microbicida e a resposta a citocinas. Os corticóides também podem ter formado complexos com receptores intracelulares que bloquearam a expressão das citocinas de linfócitos e macrófagos ${ }^{23}$.

A dexametasona age na imunidade celular e, juntamente com o antígenobase, no caso o Aspergillus fumigatus, predispõe o indivíduo às infecções. A droga passa a intervir em diversos pontos do sistema imune, prejudicando a recirculação de linfócitos (como confirmado na figura 2) e a geração de células citotóxicas efetoras ${ }^{24}$.

A significativa baixa do peso e a dificuldade de movimentar-se e locomoverse são justificáveis pelos efeitos colaterais observados pelos grupos II e IV, que utilizaram dexametasona. Os efeitos relacionam-se com a dose administrada e o período de duração. Uma única dose pode ter provocado alterações no tráfego celular (dentro de 2 horas da administração), tendo como resultado a linfopenia transitória que atinge seu pico em torno de 4 horas $^{25}$.

Com o uso da dexametasona, foi possível observar alterações nos valores hematológicos dos leucócitos, que promoveram aparente neutrofilia (cf. Tabela 1). Isso é consequiência da queda de linfócitos e monócitos no sangue, podendo ser constatado na contagem diferencial de leucócitos (cf. Tabela 2). Isso pode ter ocorrido em razão do seqüestro de linfócitos do sangue pela medula óssea e a apoptose de células T CD4. Deve ter havido liberação dos monócitos pela medula óssea, porém sem ocorrer exportação tecidual. Em relação á função celular, pode ter ocorrido a diminuição de ativação, a divisão e a proliferação dos linfócitos ${ }^{26}$.

Na figura 1, foi feita comparação do leucograma total entre os quatro grupos estudados, sendo possível observar a queda do número de leucócitos nos grupos imunossuprimidos em relação à mediana do grupo de controle. O grupo III apresentou-se com níveis leucocitários acima do grupo de controle, demonstrando ativação do sistema imune inato. Observou-se que a taxa de leucócitos do grupo

${ }^{23}$ ROINT et al, 2004.

${ }^{24}$ ROINT et al, 2003.

${ }^{25}$ CHAPEL e cols., 2003.

${ }^{26}$ ROINT et al, 2003; 2004; CHAPEL e cols., 2003.

Univ. Ci. Saúde, Brasília, v. 3, n. 2, p. 313-327, jul./dez. 2005 
IV permaneceu acima do grupo II, demonstrando que ocorreu reação imunológica, mas nada comparado à reação produzida pelo grupo III, que recebeu somente salina e fungo.

Na figura 2, observa-se sutil aumento de linfócitos do grupo IV quando comparado ao grupo II, que não recebeu o antígeno; no grupo III, esse aumento pela introdução de antígeno é exacerbada, apresentando-se acima da mediana no grupo de controle. Na figura 3, o aumento de monócitos no grupo III também é observado, existindo pequeno aumento do grupo IV quando relacionado ao grupo II.

Com isso, é possível deduzir que ocorreu ativação do sistema imune por parte do grupo IV, mas de maneira bem mais contida pela indução de imunossupressão dada no experimento, podendo comparar sua intensidade quando relacionada aos demais grupos. Pode-se constatar que o aumento de neutrófilos no hemograma diferencial ocorreu pela queda de linfócitos e monócitos e porque não houve diferença estatística quando comparado à taxa de neutrófilos dos grupos (cf. figura $5)$.

Pela análise microscópica, foi possível observar que a histologia pulmonar não demonstrou nenhum tipo de alteração patológica relacionada ao fungo, constatando-se, na leitura de lâminas coradas em HE, parênquima pulmonar normal, infiltrados linfocíticos normais, com pequeno enfisema e colabamento que podem ter sido provocados durante o manuseio do órgão. O edema e o acúmulo de hemácias nas arteríolas foram normais, visto que foi realizada hipóxia com éter para provocar a morte dos roedores. Nas lâminas complementares, coradas com grocott (coloração específica para fungos) também não foi observada presença de elementos fúngicos ${ }^{27}$.

O Aspergillus fumigatus encontra-se no grupo relacionado à maioria das mortes causadas em pacientes imunossuprimidos. Grande parte das infecções são causadas por patógenos comuns, identificados e controlados com terapia apropriada. Os microorganismos oportunistas são difíceis de isolar dificultando, assim, o diagnóstico ${ }^{28}$.

Um ponto a ser questionado pela não-obtenção do objetivo primário seria a via de inoculação; no presente trabalho, optou-se pela via intraperitonial, sendo que a via natural de infecção é intranasal. As infecções são normalmente evitadas pelas linhas de defesa como secreção de muco pelas membranas que revestem as superfícies internas do corpo. Isso pode ter gerado resistência à invasão fúngica, pois parte dos conídios inoculados podem ter sofrido rápida fagocitose de macrófagos presentes nessa região ${ }^{29}$. Maior período de ação do fungo no orga-

${ }^{27}$ ROINT et al, 2004.

${ }^{28}$ CHAPEL et al, 2003.

${ }^{29}$ TURNER et al, 1975. 
nismo com o contínuo tratamento de imunossupressão poderia gerar maior efeito patológico ao órgão, dando tempo de que o fungo se proliferasse, gerando grande efeito patogênico.

Outro fator relevante baseia-se na quantidade de conídios inoculados. Uma taxa inferior a $10^{8}$ pode ter limitado o desenvolvimento esperado, uma vez que nenhum estudo experimental da aspergilose pulmonar foi induzido com baixas concentrações de conídios. Latgé afirma que é possível diminuir a concentração de conídios quando aplicado ao modelo experimental, a imunossupressão severa, como observado em tratamento para doenças auto-imunes. É necessário que se façam modificações das questões anteriormente discutidas nas próximas pesquisas, a fim de que se obtenha modelo experimental mais eficiente, podendo, padronizar um protocolo e aterem-se a pesquisas somente os comprometimentos provocados pela patologia.

\section{Conclusão}

É de total importância que ocorram discussões e abordagens a respeito do controle das infecções pela relevância clínica e epidemiológica, a fim de que haja melhor entendimento da doença causada pelo Aspergillus fumigatus. Com a continuidade e o aprimoramento da pesquisa, será possível observar as alterações geradas por esse patógeno, contribuindo para o melhor entendimento do diagnóstico. Desta forma, é possível obter tratamento mais adequado.

A dose administrada de dexametasona mostrou-se eficaz para promover imunossupressão, mas não de maneira suficiente para oferecer condições ideais à proliferação do fungo, pois seria necessário que as taxas de neutrófilos também diminuíssem, a fim de que o fungo não fosse fagocitado. Talvez, com a substituição da dexametasona pela ciclosporina, o experimento tornasse-se mais próximo do esperado. Assim, não foi possível simular, em laboratório, a infecção gerada pelo fungo.

A biópsia é uma grande aliada ao diagnóstico da infecção, pois é possível avaliar que alterações morfológicas celulares ocorreram, permitindo diagnosticar a patologia de maneira mais específica. Chegando-se ao protocolo ideal, será possível analisar se a dose imunossupressora versus a quantidade de conídios encontram-se co-relacionados.

É importante que se dêem diagnóstico e tratamento precoce aos pacientes, em decorrência dos graves resultados quando não detectados brevemente. Em todos os pacientes imunossuprimidos, a morbidade global por infecção pulmonar geralmente excede $50 \%$.

Univ. Ci. Saúde, Brasília, v. 3, n. 2, p. 313-327, jul./dez. 2005 


\section{Referências}

ACTA MÉDICA PORTUGUESA, R.J. (ed.). Aspergilose pulmonar invasiva. vol.16, 2003. p.97-99.

ATENEU, S.P. (ed.). Tratado de anatomia sistêmica e aplicada. 27. ed., 2002. 455p .

AMORIM, D. S. et al. Infecções por Aspergillus ssp.: aspectos gerais. Revista Pulmão RJ, vol. 13, nº. p. 111-118. http://www.sopterj.com.br. [On line]. Publicado em 27 de dez. 2004.

BENGALALEGAL. Transplantes: imunossupressão e imunossupressores. http:// www.bengalalegal.com/imuno.php. [On line]. Publicado em 13 mar. 2005.

CHAPEL, H. et al.. Imunologia para o clínico. REVIER. (ed.). 4. ed 2003. Rio de Janeiro.

CLINICAL MICROBIOLOGY REVIER, (ed.). Aspegillus fumigatus and Aspergillosis. vol.12, nº. 2, 310-350p., 1999.

COBEA. Colégio Brasileiro de Experimentação Animal. 1990. http://www.cobea.org.br/ etica.htm. [On line]. Publicado em 05 de Abril. 2005.

CONTROLE E PREVENÇÃO DE INFECÇÕES. Prevenção de infecções associadas a transplantes de órgãos sólidos. http://www.cih.com.br/ preven\%C3\%A7\%C3\%A3o_em_transplantes.htm. [On line]. Publicado em 5 de janeiro de 2005.

GUANABARA KOOGAN, R. J. (ed.). Anatomia e fisiologia humana: fundamentos de anatomia clínica. Rio de Janeiro, 1998.

-_ed.). Patologia. 6. ed. 2000, p. 298-319.

-_ed.). Manual de testes diagnósticos e laboratoriais. Rio de Janeiro, 2001.

_-_ed.) Doenças pulmonares. 5. ed. Rio de Janeiro, 2002. p.442-443p.

-_ed.). Fundamentos de imunologia. 10. ed. Rio de Janeiro, 2004.

FARMÁCIA ON LINE. Farmacologia. www.farmacia.med.br/temasdesaude/ farmaco_mono_dexametasona.htm. [Online]. Publicado em 15 out. 2004.

JHAYYA, T. S.; PEREZ, D. B.; LLARGES, C. M. Bronquiolite obliterante com pneumonia em organização e aspergiloma em paciente com linfoma-leucemia de células T. In: $J$. Pneumologia, vol. 26, $\mathrm{n}^{\circ}$. 1, p. 52-54. http://www.scielo.br/scielo. php?script=sci_arttext\&pid=S0102-35862000000100011\&lng=en\&nrm= iso\&tlng=pt [On line]. Publicado em: 15 out. 2004.

LAFEPE Medicamentos. Informação sobre o medicamento Dexametasona. http:// www.lafepe.pe.gov.br/medicamentos/medicamentos. [On line]. Publicado em 13 mar. 2005.

LOPES, A. J.et al Aspergilose pulmonar. In: Revista Pulmão RJ, vol. 13, no.1, p. 34-44. http:/ /www.sopterj.com.br. [On line]. Publicado em 02 jan. 2005.

Manual Merck: Diagnóstico e Tratamento - Edição Centenária. Distúrbios dos Pulmões e das vias aéreas: Pneumonia. http://www.msd-brazil.com/ msd43/ m_manual/ 
mm_sec4_41.htm. [On line]. Publicado em 16.10.2004.

MARCHIORI, E., VALIANTE, P.M.; SOUZA JÚNIOR.; A.S. Nódulos com sinal do halo na aspergilose pulmonar angioinvasiva: correlação da tomografia computadorizada de alta resolução com a anatomopatologia. In: Radiol Bras, vol.35, nº . 4. p.195-198. http://www.scielo.br/scielo.php?script=sci_abstract\&pid= S010039842002000400003\&lng=en\&nrm=iso\&tlng=pt . [On line]. Publicado em 20 out. 2004.

MARCHIORI, E. et al. Granulomas hialinizantes pulmonares: aspectos na tomografia computadorizada: relato de caso. In: Radiol Bras, vol. 36, nº. 6, p.385-387. http:// www.scielo.br/scielo.php?script=sci_abstract\&pid=S0100-39842003000600011 \&lng=en\&nrm= iso\&tlng=pt [On line]. Publicado em 21 out. 2004.

MICROBIOLOGIANAINTERNET. Bacilos gran negativos não fermentadores. http:// www.microbiologianainternet.hpg.ig.com.br/bgnnf.htm [On line]. Publicado em:20 fev. 2005.

NEUFELD, P. M. Aspergilose pulmonar: aspectos histopatológicos, clínicos e radiológicos. In: SBAC Jornal, $\mathrm{n}^{\circ} .20$ março, p. 30-31, 2004.

NEVES V.; FANICA. M. J. Novos antifúngicos. In: Revista Ordem dos Farmacêuticos. n ${ }^{\circ}$ 59,p.12.http://www.ordemfarmaceuticos.t/frontoffice/pages/ defaultCategoryViewOne.asp?catId=258 [On line]. Publicado em 04 dez. 2005.

SARVIER, S. P. (ed.). Tratado de micologia médica lacaz. 9. ed. 2002, 1105 p.

SAÚDE BRASIL. Efeito do Glicocorticóide Dexametasona sobre proliferação de células $9 \mathrm{~L}$ de Glionarcoma Murino. www.saudebrasilnet.com.br/premios/oncologia/premio1/ trabalhos/009c.pdf. [On line]. Publicado em 22 mar. 2005.

WEBCIÊNCIA. Corpo humano: pulmão. São Paulo, maio de 2000. http:// www.webciencia.com/11_18pulmao.htm. [On line]. Publicado em 15 out. 2004. 


\section{Envie suas publicações para o Conselho Editorial} da revista Universitas - Ciências da Saúde.

Endereço para envio: SEPN 707/907, Campus do UniCEUB 70790-075 Brasília-DF

Fone: (61) 3340-1397

$$
\text { universitas.saude@uniceub.br }
$$

Verifique as normas de submissão no final deste número 